\title{
In vivo distribution and antitumor effect of infused immune cells in a gastric cancer model
}

\author{
XIAOHUI DU ${ }^{1,2}$, RUNSEN JIN ${ }^{2,3}$, NING NING ${ }^{1,2}$, LI LI $^{2}$, QUANSHENG WANG ${ }^{2}$, \\ WENTAO LIANG ${ }^{2}$, JUCHAO LIU ${ }^{2}$ and YINGXIN XU ${ }^{2}$ \\ ${ }^{1}$ General Surgery Department, Hainan Branch of Chinese PLA General Hospital, Sanya, Hainan; \\ ${ }^{2}$ Institute of General Surgery, Chinese PLA General Hospital, Beijing; ${ }^{3}$ General Surgery Department, \\ Rui Jin Hospital, School of Medicine, Shanghai Jiao Tong University, Shanghai, P.R. China
}

Received May 14, 2012; Accepted June 13, 2012

DOI: $10.3892 /$ or.2012.2013

\begin{abstract}
Adoptive cellular transfer has been employed for cancer immunotherapy, including patients with gastric cancer. However, little is known about the distribution of effector cells after their injection via different pathways. In this study, we used human gastric cancer cells (BGC823) tagged with enhanced green fluorescent protein (EGPF) to establish a subcutaneous gastric cancer model in nude mice. Cytokineinduced killer (CIK) cells and cytotoxic T lymphocytes (CTLs) were generated from human peripheral blood and labeled with red fluorescent PKH26. A portion of CIK cells was armed with CEA/CD3-bispecific single-chain antibody. When CIK cells were injected into nude mice with established subcutaneous gastric cancer via peritumoral (p.t.), intravenous (i.v.) and intraperitoneal (i.p.) infusion respectively, the distribution of cells was observed using a live fluorescence imaging system. We found that only a very small number of CIK cells could travel to the tumor site after i.p. or i.v. infusion, and they inhibited subcutaneous tumor growth in vivo only immediately following injection. In contrast, p.t. injection resulted in a significantly higher accumulation of CIK cells at the tumor site for 48 hours and mediated the greatest tumor inhibition compared with the other two injection methods. In addition, we compared the antitumor activity of CIK, CEA/ CD3-bscAb-CIK and CTL cells in vitro and in vivo after p.t. injection. Among the three types of immune cells, CTLs demonstrated the strongest antitumor activity both in vitro and in vivo. CEA/CD3-bispecific single chain antibody could effec-
\end{abstract}

Correspondence to: Dr Xiaohui Du or Dr Yingxin Xu, Institute of General Surgery, Chinese PLA General Hospital, No. 28 Fuxing Road, Haidian District, Beijing 100853, P.R. China

E-mail: duxiaohui301@163.com; xuyingxin301@yeah.net

Key words: gastric cancer model, adoptive cellular immunotherapy, CIK cells, CTL cells, bispecific antibody, antitumor activity, in vivo distribution tively link T lymphocytes and tumor cells expressing CEA, and resulted in significantly higher accumulation of CIK cells at the tumor site compared with the parental CIK cells. This study indicates that peritumoral injection of immune effector cells by minimally invasive surgical procedures represents an effective delivery method of adoptive cellular immunotherapy. Tumor-specific immune cells, such as CTLs, are a better choice of effector cells than CIKs in cellular immunotherapy. Furthermore, $\mathrm{CD}^{+}$immune cells armed with the CEA/ CD3-bispecific single chain antibody could more effectively travel to and accumulate at the site of tumors expressing CEA, such as gastric cancer.

\section{Introduction}

Gastric cancer is the fourth most common cancer in the world (1). Standard methods of treatment, e.g. surgery, chemotherapy and radiotherapy have shown mixed success for early-stage patients. However, the prognosis of patients with advanced-stage gastric cancer is still extremely poor. Currently, transfusion of lymphocytes or adoptive cellular immunotherapy (ACI) have been used for the treatment of cancer both in animal studies (2-4) and clinic trials (5-7). ACI has also been used to treat gastric cancer with encouraging results, indicating that ACI can improve the prognosis of some patients, especially those who cannot tolerate surgery (8).

Cytokine-induced killer cells (CIKs) are commonly used as immune effector cells to treat cancer (9-11). The major component of the heterogeneous CIK cells, NKT cells, expresses both $\mathrm{T}$ cell marker CD3 and NK cell marker CD56 and exhibits non-major histocompatibility complex (MHC)restricted cytotoxicity. This represents an effective mechanism in the treatment of cancer. Infusion of CIK cells is typically through an intravenous pathway; however, little is known regarding the number of cells that actually arrive at the tumor site with this method and how long these cells could survive in vivo.

Apart from CIK cells, many other immune cells such as lymphokine-activated killer (LAK) cells, tumor infiltrating lymphocytes (TILs) and antigen-specific cytotoxic T lymphocytes (CTLs) have been used in adoptive therapy (5). CTLs 
play a central role in antitumor immunity $(12,13)$ and have demonstrated therapeutic efficacy of cancer immunotherapy both in vivo and in vitro. However, the in vivo distribution of these cells following various methods of injection is also unclear.

In this study, we infused PKH26-labeled human CIK cells or CTLs into nude mice with established EGFP-positive human gastric cancer through different pathways and sequentially examined the tissue distribution of CIK cells and CTLs using live fluorescence imaging. This study intended to identify the effective ACI cell type as well as the effective route of immune cell delivery to the tumor.

\section{Materials and methods}

Mice. Four-week-old female BALB/c nude (nu/nu) mice were acquired from the Animal Center of the Academy of Military Medical Sciences (Beijing, China). All animals were maintained in a pathogen-free environment and all animal protocols followed the experimental procedures of the National Institutes of Health Guide for Care and Use of Laboratory Animals.

Cell line. The poorly differentiated human gastric adenocarcinoma cell line, BGC823, was purchased from the Chinese Academy of Medical Sciences (Beijing, China). These cells are kept in our laboratory at the Institute of General Surgery, General Hospital of PLA (Beijing, China) and maintained in Dulbecco's modified Eagle's medium (DMEM) (Sigma, St. Louis, MO, USA).

DNA transfection and isolation of stable EGFP-expressing cells. BGC823 tumor cells were transfected with the pEGFP-C1 plasmid (Clontech, Mountain View, CA, USA) using the $\mathrm{Xfect}^{\mathrm{TM}}$ transfection reagent (Clontech). The neomycin resistance gene (neoR) in pEGFP-C1 plasmid allows stably transfected tumor cells to be selected using G418. After transfection, the cells were passaged at a ratio of 1:5 in selective medium that contained $200 \mu \mathrm{g} / \mathrm{ml}$ geneticin (G418; Sigma). The level of G418 was increased to 2,000 $\mu \mathrm{g}$ / $\mathrm{ml}$ in a stepwise manner (increased every day by $100 \mu \mathrm{g} / \mathrm{ml}$ ). Cell clones expressing high levels of EGFP were isolated by limit dilution in 96-well plates. The EGFP-expressing clones were then amplified and transferred by conventional culture methods. This resulted in the identification of a cell line with bright EGFP fluorescence, designated as BGC823-EGFP that was used in this study.

Tumor challenge. Nude mice were challenged with $1 \times 10^{7}$ BGC823-EGFP cells, subcutaneously. Whole-body images were taken using the IVIS-200 Imaging System (Xenogen, Alameda, CA, USA) on Day 10.

Dendritic cell isolation and culture. Peripheral blood mononuclear cells (PBMCs) from healthy donor were cultured with Cellix-901 medium (Beijing XinMingLiTai Bio-technique, Co., Ltd., Beijing, China) for $2 \mathrm{~h}$. Adherent cells were collected and further cultured in Cellix-901 medium with 1,000 IU/ml rhIL-4 (Cell Genix) and 1,000 IU/ml rhGM-CSF for 7 days to generate a dendritic cell (DC)-enriched cell population. DCs were then pulsed with $3 \mu \mathrm{l} / \mathrm{ml}$ Pseudomonas aeruginosa and
$20 \mu \mathrm{g} / \mathrm{ml}$ BGC823 cell lysate followed by incubation for $12 \mathrm{~h}$ before use for the next step. To prepare BGC823 cell lysate, BGC823 cells were digested with trypsin, washed in normal saline and centrifuged at $450 \mathrm{x} \mathrm{g}$ for $5 \mathrm{~min}$. The supernatant was then discarded and cells were resuspended in sterile water, and were frozen and thawed repeatedly 3 times. Lysate was obtained after centrifugation and filtration.

CIK, CTL and CIK-CEA/CD3-bscAb cell isolation and culture. Using a blood cell separator (Institute of Biomedical Engineering, Chinese Academy of Medical Sciences), $2-4 \times 10^{9}$ PBMCs from healthy donors were obtained. To prepare CIK, cell concentration was adjusted to $2 \times 10^{6}$ cells/ $\mathrm{ml}$ in fresh, serum-free Cellix-601 medium (Beijing XinMingLiTai Bio-technique, Co., Ltd.) with 2,000 U/ml rhIFN- $\gamma$ and incubated at $37^{\circ} \mathrm{C}$ in a humidified atmosphere of $50 \mathrm{ml} / 1 \mathrm{CO}_{2}$. After $24 \mathrm{~h}$, anti-CD3 (50 ng/ml) and rhIL-2 $(1,000 \mathrm{U} / \mathrm{ml})$ were added. On Days $0,4,7$ and 10 of culture, cell densities were determined and phenotypes were identified by flow cytometric analysis (FACS) (Becton-Dickinson, USA). To make CTL cells, PBMCs were incubated in tissue culture flasks with the Ag-pulsed DCs. To generate CIK-CEA/ CD3-bscAb cells, CEA/CD3-bispecific single chain antibody (Beijing ABT Genetic Engineering Technology, Co., Ltd., Beijing, China) was added into the medium to bind CIK cells to obtain CIK-CEA/CD3-bscAb cells.

Immune cell labeling and in vitro assays. Immune cells were labeled with red fluorescent PKH26 (Sigma-Aldrich, Co., LLC.) following the kit instructions. CIK-, CIK-CEA/ CD3-bscAb- and CTL-mediated cell cytotoxicity was evaluated using the lactate dehydrogenase $(\mathrm{LDH})$ release assay according to the manufacturer's protocol. Cytotoxicity was assessed as $\left[\left(\mathrm{A}_{\text {sample }}-\mathrm{A}_{\text {spontaneous }}\right) /\left(\mathrm{A}_{\text {maximum }}-\mathrm{A}_{\text {spontaneous }}\right)\right] \times 100 \%$, (A, absorbance).

Tracing immune cells in vivo and evaluating the inhibition of tumor growth. Seven days after BGC823-EGFP cells were implanted into the nude mice, the tumor-bearing animals were separated into 3 groups. All mice were injected with $1 \times 10^{7}$ labeled CIKs, intraperitoneally (i.p., Group 1) ; intravenously (i.v., Group 2) or peritumorally (p.t., Group 3), respectively. The migration and distribution of the infused CIK cells were then observed using the IVIS-200 Imaging System (Xenogen) at 4, 24, 48 and $96 \mathrm{~h}$ after CIK cells injection. The lung, liver, spleen, kidney, stomach and intestine were collected on Days 2 and 8 for histopathological analysis. Tumor size and weight were assessed on Day 35 to evaluate the therapeutic efficacy of transferred CIK cells.

In separate experiments, we set up 3 groups to compare the therapeutic efficacy of CIK, CIK-CEA/CD3-bscAb and CTL cells. Each group of labeled immune cells was injected peritumorally at $1 \times 10^{7}$ cells/mouse. Migration and distribution of the infused immune cells were then observed at 4, 24, 48, 96 and $144 \mathrm{~h}$ after cell infusion.

Statistical analysis. Statistical analyses were performed with the SPSS 17.0 statistical software using the Student's t-test and ANOVA. P-values of $<0.05$ were considered statistically significant between the experimental groups. 
Table I. In vitro antitumor effects of CIK cells before and after labeling with PKH26 (mean $\pm \mathrm{SD}, \mathrm{n}=3$ ).

\begin{tabular}{lcccccc}
\hline & & \multicolumn{2}{c}{$5 \mathrm{~h}$} & & \multicolumn{2}{c}{$10 \mathrm{~h}$} \\
\cline { 3 - 4 } \cline { 5 - 6 } Groups & E/T ratio & LDH & Cytotoxicity, $\%$ & & LDH & Cytotoxicity, $\%$ \\
\hline CIK & $5: 1$ & $0.457 \pm 0.023$ & $70.51 \pm 3.61$ & & $0.555 \pm 0.024$ & $95.43 \pm 4.84$ \\
& $10: 1$ & $0.697 \pm 0.081$ & $84.44 \pm 4.48$ & & $0.894 \pm 0.090$ & 100 \\
CIK-PKH26 & $5: 1$ & $0.460 \pm 0.017$ & $68.88 \pm 3.67$ & & $0.555 \pm 0.023$ & $93.78 \pm 3.06$ \\
& $10: 1$ & $0.701 \pm 0.063$ & $83.54 \pm 4.98$ & & $0.901 \pm 0.028$ & 100 \\
\hline
\end{tabular}

LDH, lactate dehydrogenase release assay; CIK, cytokine-induced killer cells.

Table II. Antitumor effect of different immune cells in vitro (mean $\pm \mathrm{SD}, \%, \mathrm{n}=3$ ).

\begin{tabular}{|c|c|c|c|c|}
\hline \multirow[b]{2}{*}{ Groups } & \multicolumn{2}{|c|}{$5 \mathrm{~h}$} & \multicolumn{2}{|c|}{$10 \mathrm{~h}$} \\
\hline & $5: 1 \mathrm{E} / \mathrm{T}$ ratio & 10:1 E/T ratio & $5: 1 \mathrm{E} / \mathrm{T}$ ratio & 10:1 E/T ratio \\
\hline CIK & $70.51 \pm 3.61$ & $84.44 \pm 4.48$ & $95.43 \pm 4.84$ & 100 \\
\hline CIK-CEA/CD3-bscAb & $74.09 \pm 2.01$ & $88.01 \pm 2.88$ & $100^{\mathrm{c}}$ & 100 \\
\hline CTL & $85.59 \pm 5.14^{\mathrm{a}, \mathrm{b}}$ & $97.47 \pm 2.20^{\mathrm{a}, \mathrm{b}}$ & $100^{\mathrm{c}}$ & 100 \\
\hline
\end{tabular}

${ }^{\mathrm{a}} \mathrm{P}<0.05$ vs. the CIK group; ${ }^{\mathrm{b}} \mathrm{P}<0.05$ vs. the CIK-CEA/CD3-bscAb group; ${ }^{\mathrm{c}} \mathrm{P}<0.01$ vs. the $\mathrm{CIK}$ group. CIK, cytokine-induced killer cells; $\mathrm{CTL}$, cytotoxic $\mathrm{T}$ lymphocytes.

A

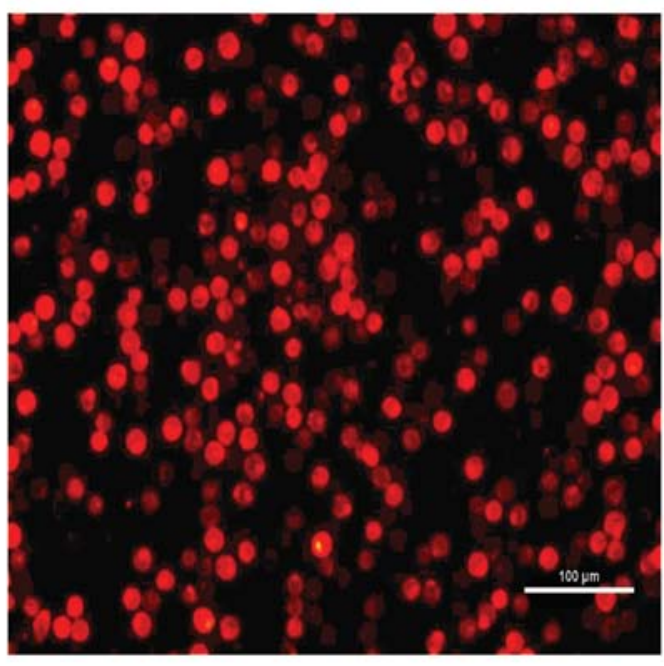

B
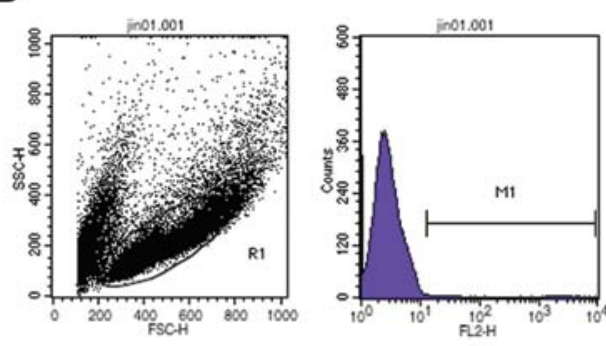

Histogram Statisices

File: in01.001

Sample ID: contec

$\frac{\text { Marker } \% \text { Gated }}{\text { Al } 100.00}$

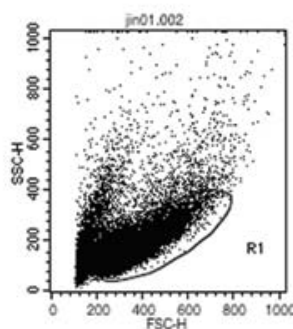

Figure 1. CIK cells after labeling with PKH26. (A) Strong red fluorescence was detected with fluorescence microscopy after labeling; (B) FACS analysis showed that $99.37 \%$ of CIK cells were successfully labeled with PKH26.

\section{Results}

Antitumor effect of different immune cells in vitro. Firstly, we detected the antitumor effect of labeling on the immune cells. No difference was observed in cell morphology after labeling with PKH26. Strong red fluorescence was detected by flxuorescence microscopy (Fig. 1A) and FACS analysis showed that $99.37 \%$ of CIK cells were PKH26-positive (Fig. 1B).
There was no significant difference in the antitumor activity between CIK and CIK-PKH26 cells (Table I).

In addition to CIK cells, CIK-CEA/CD3-bscAb cells and CTLs also showed strong antitumor activity against BGC823 cells in vitro. At E:T cell ratios of 5:1 and 10:1 and at 5 and $10 \mathrm{~h}$, the BGC823 cell killing activity was as follows: CTL > CIK-CEA/CD3-bscAb > CIK cells (Table II). 
A

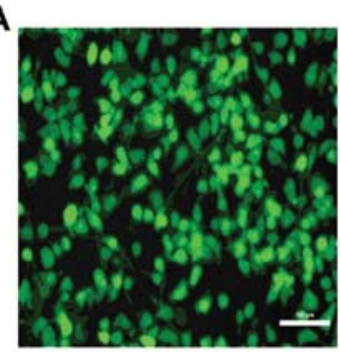

C

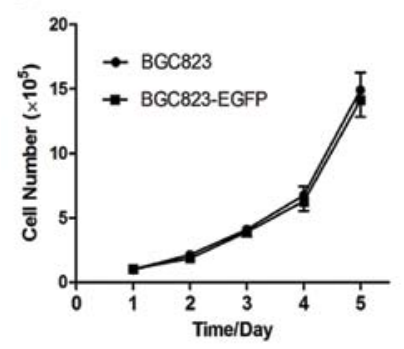

E

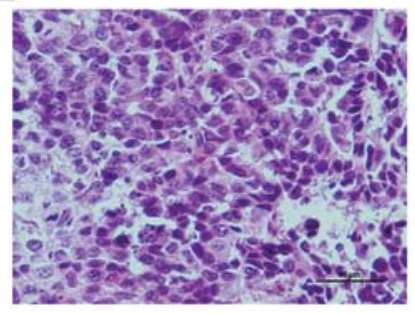

B

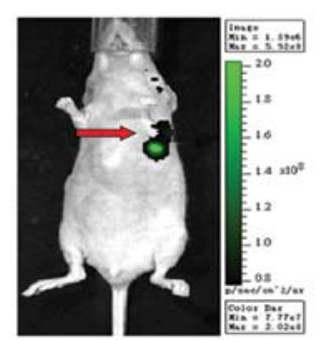

D

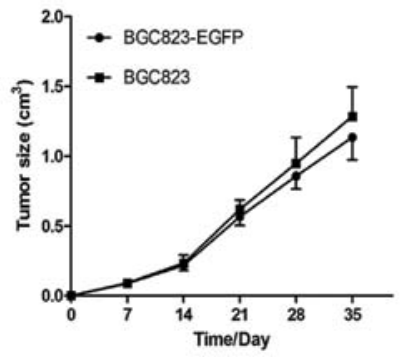

$\mathbf{F}$

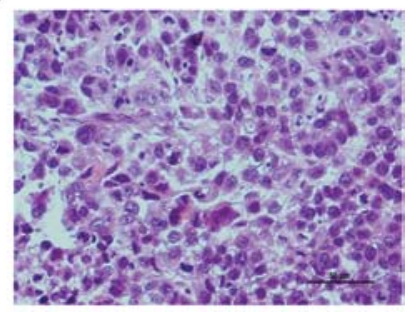

Figure 2. The human gastric cancer cell line BGC823 was transduced with the pEGFP-C1 plasmid using the Xfect transfection reagent, resulting in a high-level of EGFP expression. (A) Strong green fluorescence was detected with fluorescence microscopy; (B) whole-body image of the nude mouse injected with BGC823-EGFP tumor cells subcutaneously. Picture was taken by IVIS-200 Imaging System and arrow points to the tumor. (C) No difference in in vitro growth between BGC823 and BGC823-EGFP was observed over 5 days; (D) no difference in in vivo growth between BGC823 and BGC823-EGFP was observed over 5 weeks; (E) H\&E slide of BGC823 subcutaneous tumor (magnification, x200); (F) H\&E slide of BGC823-EGFP subcutaneous tumor (magnification, $\mathrm{x} 200$ ).

Establishment of human gastric cancer (BGC823) in nude mice subcutaneously. Fluorescence imaging was employed to monitor the growth of human gastric cancer in nude mice. First of all, we successfully transfected BGC823 cells with EGFP (BGC823-EGFP cells) and selected them with G418 (Fig. 2A). We then injected parental BGC823 and BGC823EGFP cells subcutaneously into the nude mice, respectively. The BGC823-EGFP subcutaneous tumor was visible with the IVIS-200 Imaging System within 10 days after tumor cell implantation (Fig. 2B).

EGFP transfection was found to have no effect on BGC823 cell morphology and cell proliferation in vitro (Fig. 2C) or tumor growth in vivo (Fig. 2D). In addition, no difference was observed in the histopathological analysis between BGC823 and BGC823-EGFP tumors (Fig. 2E and F).

Distribution and antitumor effect of CIK cells infused via three different pathways. When CIK cells were i.p. injected (Fig. 3A), red fluorescent PKH26-labeled CIK cells tended to gather first in the abdominal cavity. At $24 \mathrm{~h}$ post-injection, cells began to spread, and a small amount of accumulation could be observed

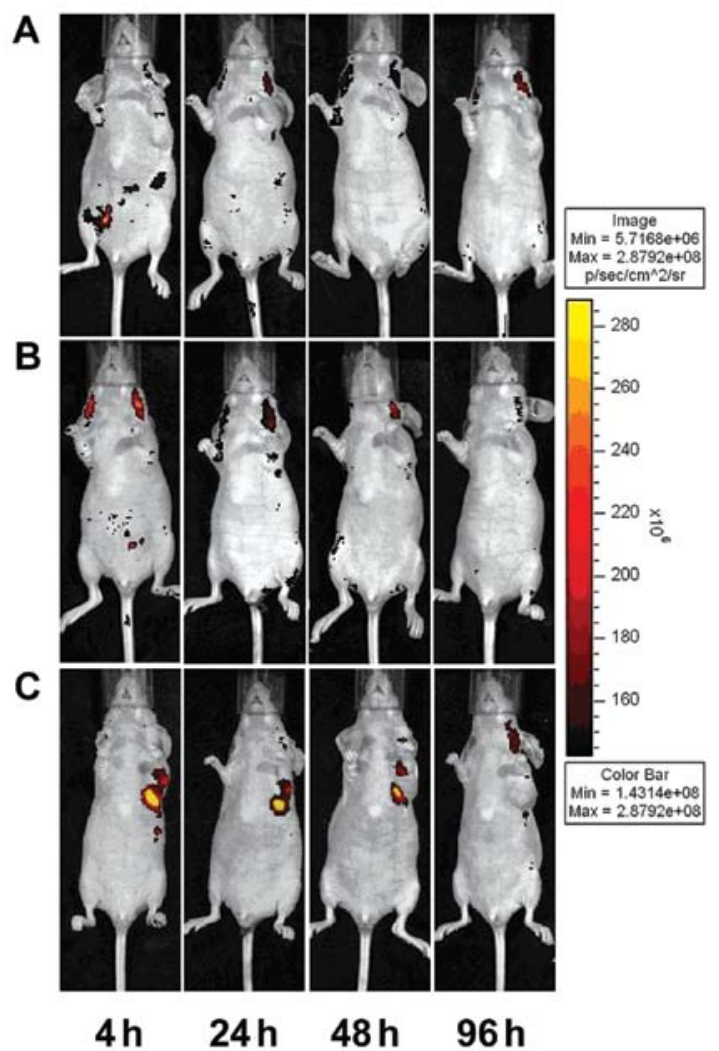

Figure 3. The distribution of CIK cells after infusion into the tumor-bearing nude mice; (A) i.p., (B) i.v. and (C) peritumoral injection groups.

in the tumor area before gradually dissipating. On Day 5 post-injection, the red fluorescence was nearly gone. In the i.v. group (Fig. 3B), CIK-PKH26 cells dispersed rapidly in the body, and a small amount of accumulation could be observed in the tumor area $24 \mathrm{~h}$ post-injection. On Day 5 , the red fluorescence vanished. In contrast, in the peritumoral injection group (Fig. 3C), CIK-PKH26 cells gathered around the tumor after injection and dissipated very slowly. On Day 5, we could still detect a small amount of red fluorescence around the tumor.

On Day 2 post-injection via p.t. injection, labeled CIK cells were found to infiltrate the tumor bed in high numbers, although this infiltration was mainly localized around the tumor (Fig. 4A), while in the i.p. (Fig. 4B) and i.v. groups (Fig. 4C), the red fluorescent spots were much fewer. In the i.p. group, the labeled CIK cells were detected infiltrating multiple organs. The spleen was the most enriched in CIK cells (Fig. 4D), followed by the liver (Fig. 4E), kidney (Fig. 4F), intestine (Fig. 4G), stomach (Fig. 4H) and lung (Fig. 4I). On Day 8 post-injection, we found only a small quantity of red fluorescent spots in the liver and spleen in the i.p. and i.v. groups; however, in the peritumoral injection group, some labeled CIK cells were still found infiltrating the tumor bed (Fig. 4J), liver (Fig. 4K) and spleen (Fig. 4L).

The infusion of CIK cells via each of the three pathways, particularly peritumorally, was found to inhibit subcutaneous tumor growth in nude mice. Three weeks after CIK transfusion, tumors of the peritumoral injection group were significantly smaller than the normal saline (NS)-treated control group. Although not statistically significant, tumors of the i.p. and i.v. groups were also smaller. Four weeks after transfusion, 
A

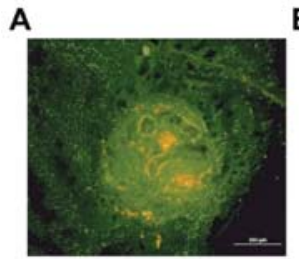

B

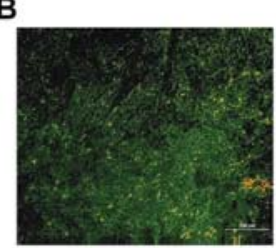

D
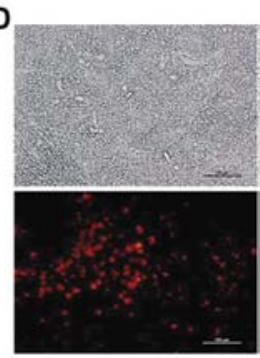

E
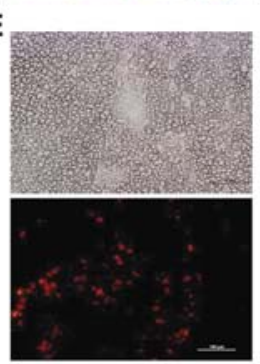

G
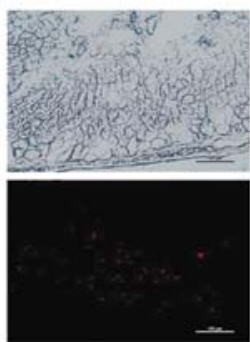

H
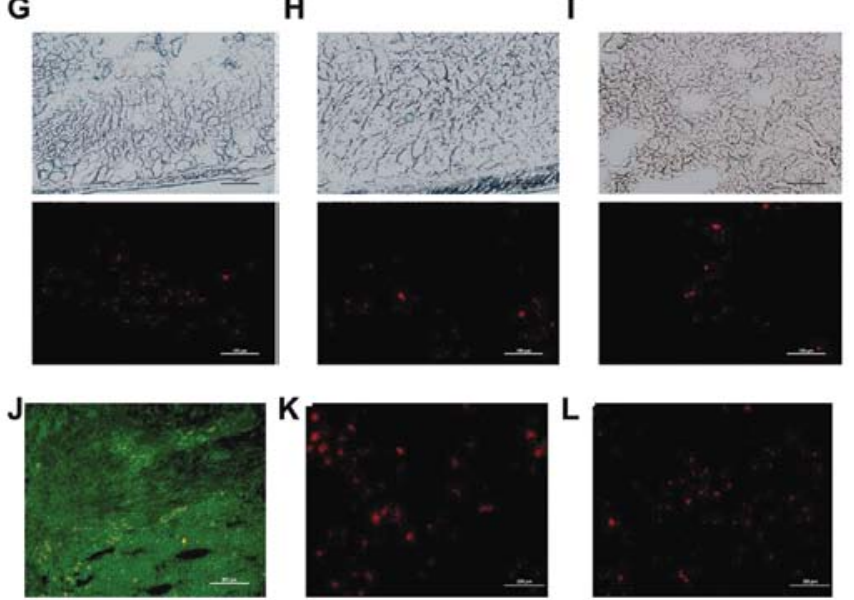

Figure 4. Frozen sections of tumors. (A) Peritumoral injection, (B) i.v. and (C) i.p. groups on Day 2. (D) Spleen, (E) liver, (F) kidney, $(\mathrm{G})$ intestines, $(\mathrm{H})$ stomach and (I) lung on Day 2. (J) Tumor, (K) liver and (L) spleen on Day 8.

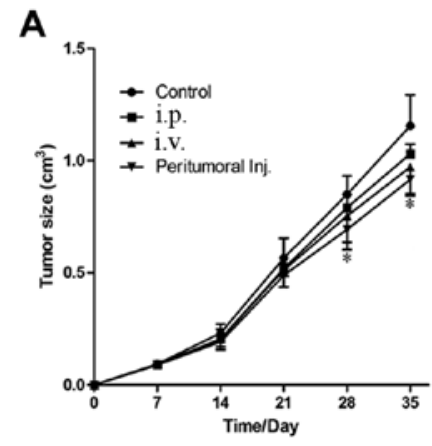

B

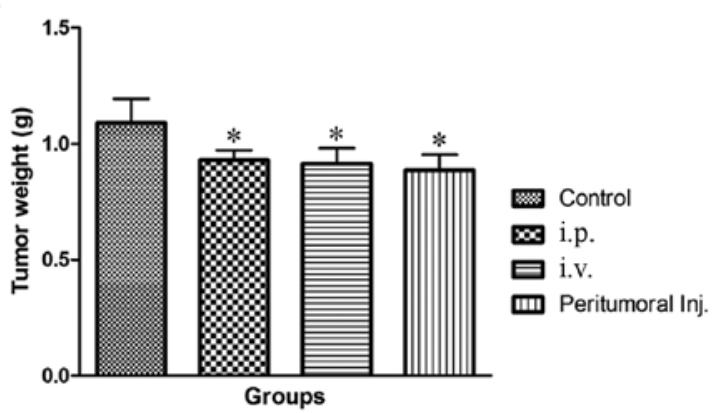

Figure 5. Tumor size and weight after the infusion of CIK cells via different pathways as indicated. Statistical significance was determined using the Student's t-test vs. the NS-treated control group $\left(n=4,{ }^{*} \mathrm{P}<0.05\right)$.

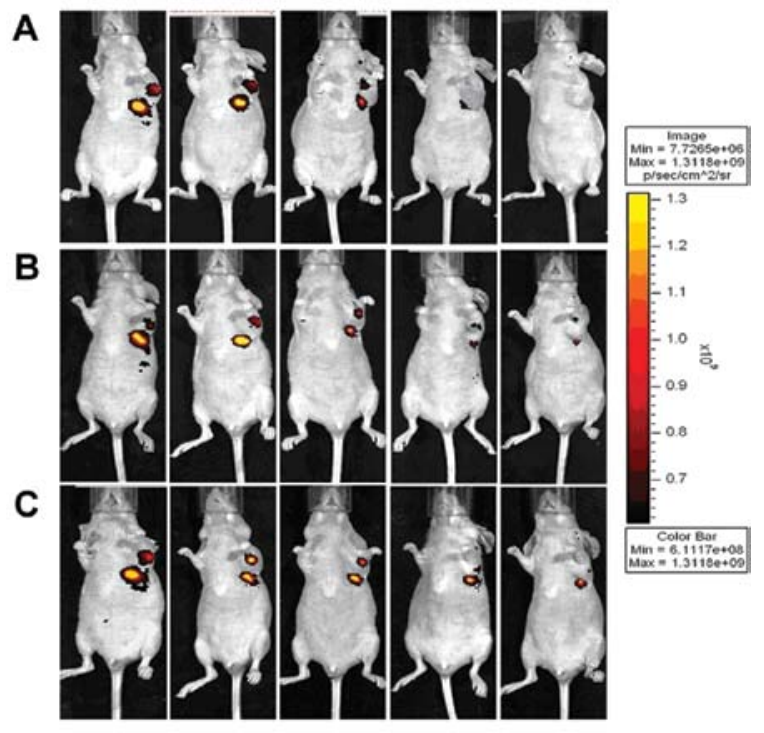

\section{4h $24 h \quad 48 h \quad 72 h \quad 144 h$}

Figure 6. The distribution of different immune cells after peritumoral injection. (A) CIK, (B) CTL and (C) CIK-CEA/CD3-bscAb groups.

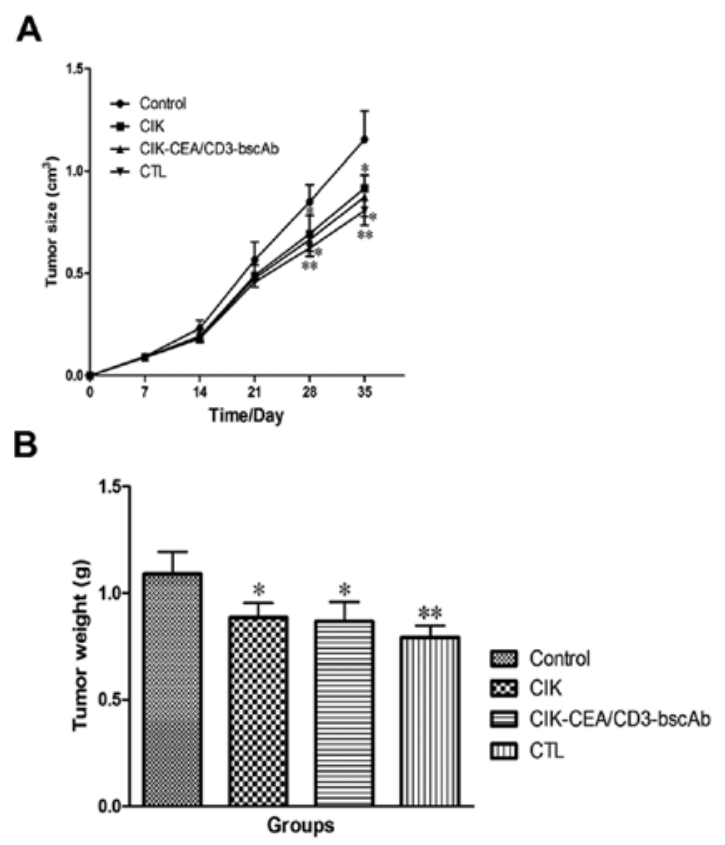

Figure 7. Tumor size and weight after the infusion of 3 different immune cells via peritumoral injection. Statistical significance was determined using the Student's t-test vs. the NS-treated control group $\left(\mathrm{n}=4,{ }^{*} \mathrm{P}<0.05,{ }^{* *} \mathrm{P}<0.01\right)$.

tumor weight in the peritumoral, i.v. and i.p. injection groups was significantly lighter than the NS-treated control groups (Fig. 5).

Distribution and antitumor effect of different immune cells infused via peritumoral injection. Following peritumoral injection, all of the immune cells were gathered around the tumor. CIK cells were nearly gone 5 days post-injection (Fig. 6A), followed by the dispersal of CTL cells within 7 days (Fig. 6B). However, CIK-CEA/CD3-bscAb cells remained crowded around the tumor 7 days post-injection (Fig. 6C). 
Peritumoral injection of three immune cell types, particularly CTLs, was found to inhibit subcutaneous tumor growth in nude mice (Fig. 7). Control tumor weight was $1.09 \pm 0.10 \mathrm{~g}$ 35 days after implantation. CTL, CIK-CEA/CD3-bscAb and CIK cells injected peritumorally 7 days after tumor implantation reduced tumor weight by $27.29 \pm 5.05,20.41 \pm 8.35$ and $18.58 \pm 6.01 \%$, respectively.

\section{Discussion}

Whole-body imaging has proven to be a useful technology for the study of the dynamics of metastatic cancer. Green fluorescent protein (GFP) expression in cancer cells can help externally to image and follow the natural course or impediment of tumor progression and metastasis (14). Tumor motility, progression and metastasis can be visualized at the single-cell level in vivo with GFP (15). EGFP is the enhanced GFP, which is made for a heightened brightness (16). In this study, we have successfully established the stable EGFP-expressing human gastric adenocarcinoma BGC823 cells (BGC823-EGFP). These cells form subcutaneous tumors in nude mice. BGC823EGFP cells have revealed identical biological characteristics as parental BGC823 cells, and can therefore be used to investigate tumor growth and metastasis $(15,17,18)$.

CIK cells are polyclonal $\mathrm{T}$ effector cells generated when cultured under conditions of cytokine stimulation (19). The main effector cells of the heterogeneous CIK cell family express NK and T cell markers CD56 and CD3, respectively, and are referred to as NK-like T (NKT) cells. These cells possess non-MHC-restricted antitumor activity which means they do not require prior specific sensitization to induce the recognition of target cells $(9,20,21)$. Over the years, CIK cells have been used for their antitumor activity against a variety of tumor targets $(10,11,22)$. In this study, we found that CIK cells killed $100 \%$ of BGC823 cells within $10 \mathrm{~h}$ at the E:T cell ratio of 10:1 in vitro. CIK cells also exhibited antitumor activity in vivo. In our gastric cancer nude mouse model, infused CIKs inhibited subcutaneous tumor growth. Importantly, we observed that CIK cells infused via different pathways demonstrated different distribution and tumor inhibitory effect.

CIK cells are usually infused intravenously in adoptive cellular immunotherapy (ACI). Hazelrigg et al (23) found that the immune cells first arrived at the lungs after intravenous transfusion. In 2-6 h, accumulation in the lungs reached a peak and dissipated, with gradual accumulation in the liver, kidney and spleen. The overall cell distribution tended to stabilize within 24 h. Skitzki et al (24) reported that CIK cells could widely immigrate into most organs after intravenous transfusion; distribution was related to blood supply and immune properties of the organs as well as the order in which cells reached each organ. Furthermore, the in vivo cytotoxic activity of adoptively transferred immune cells is mainly observed in the initial peak period post-transfusion. These studies suggest that immune cells can effectively reach most organs and tumor tissue while maintaining their cytotoxic abilities. For leukemia, lymphoma and other non-solid tumors, CIK cells can spread throughout the body, including the bone marrow, via intravenous transfusion to kill tumor cells $(25,26)$. However, for solid tumors, such as gastric cancer, the ability of CIKs to target and accumulate in tumor tissue remains to be fully defined.
In our study, we found that, after intravenous transfusion, CIK cells dissipated with the blood circulation and could indeed arrive at the tumor tissue. In comparison, intraperitoneally infused CIK cells first gathered in the abdominal cavity, then distribution followed the same course as the intravenous group by $24 \mathrm{~h}$. In contrast, peritumoral injection resulted in the maintenance of CIK cells in the tumor tissue for the maximum amount of time examined. In this case migration of infused CIK cells to other organs, such as the liver and spleen, was evident but not to the degree of the i.v. or i.p. groups. In terms of tumor growth, peritumoral injection of CIK cells showed improved inhibition. Together, these results indicate that immune cells used for cancer adoptive immunotherapy, if injected directly into the tumor area, may be able to achieve the maximum antitumor effect.

In order to potentiate the antitumor activity of CIK cells, many immunological manipulations have been developed. For example stimulating factors such as Bacille CalmetteGuerin (27) and virus vaccine (28) were added in cell culture to improve cell proliferation. Interleukin-2 (IL-2) genes were transfected into CIK cells to enhance their IL-2 production and potentiate their cytotoxicity (29). DCs were engineered to present tumor antigens to CIK cells with the hopes of enhancing specific recognition of tumor cells and their subsequent killing $(30,31)$. Furthermore, bispecific antibodies for CD3 on T and/or NKT cells and surface antigen on the target cells were used to promote the engagement of CIK cells with target cells $(32,33)$. We have previously found that the gastric adenocarcinoma BGC823 cells express surface carcinoembryonic antigen (CEA). We therefore used CEA/CD3-bispecific single chain antibody to coat CIK cells. CEA/CD3-bscAb can pull together $\mathrm{T}$ lymphocytes and CEA-expressing tumor cells (34). In this study, CIK-CEA/CD3-bscAb cells showed stronger antitumor activity than CIK cells against BGC823 cells in vitro. Use of CEA/CD3-bscAb to arm CIK cells may represent a potential approach to enhance the antitumor activity of CIK cells.

CTL cells are a subpopulation of T cells with specific cytotoxicity. $\mathrm{CD}^{+} \mathrm{CTLs}$ are the most numerous members of the CTL subgroup. After priming by antigen presented by antigen presenting cells, CTLs can recognize and kill corresponding target cells $(35,36)$. Adoptive transfer of antitumor CTLs has already been employed in clinical trials and has shown to be effective in adoptive immunotherapy of ovarian cancer, melanoma, breast cancer and renal carcinoma (12,37-40). In this study, CTL cells were derived from PBMCs and stimulated by DCs loaded with BGC823 tumor cell lysate. We found that the antitumor activity against gastric cancer of the CTLs was much stronger than CIK cells not stimulated with Ag-loaded DCs.

In summary, our results indicate that immune cells such as CIKs and CTLs have strong and direct cytotoxic effects on gastric cancer cells, which suggests that immunotherapy using these immune cells, may become a novel treatment strategy for patients with gastric cancer following surgery, radiotherapy and chemotherapy.

\section{Acknowledgements}

This study was supported by the Natural Science Foundation of China (no. 60601018). 


\section{References}

1. Kamangar F, Dores GM and Anderson WF: Patterns of cancer incidence, mortality, and prevalence across five continents: defining priorities to reduce cancer disparities in different geographic regions of the world. J Clin Oncol 24: 2137-2150, 2006.

2. Kim HM, Kang JS, Lim J, et al: Antitumor activity of cytokineinduced killer cells in nude mouse xenograft model. Arch Pharm Res 32: 781-787, 2009.

3. Klebanoff CA, Gattinoni L, Palmer DC, et al: Determinants of successful $\mathrm{CD}^{+} \mathrm{T}$-cell adoptive immunotherapy for large established tumors in mice. Clin Cancer Res 17: 5343-5352, 2011.

4. Marcus A and Eshhar Z: Tumor-specific allogeneic cells for cancer therapy. Expert Opin Biol Ther 11: 1551-1554, 2011.

5. June $\mathrm{CH}$ : Adoptive $\mathrm{T}$ cell therapy for cancer in the clinic. J Clin Invest 117: 1466-1476, 2007.

6. Di Stasi A, Tey SK, Dotti G, et al: Inducible apoptosis as a safety switch for adoptive cell therapy. N Engl J Med 365: 1673-1683, 2011.

7. Zhong JH, Ma L, Wu LC, et al: Adoptive immunotherapy for postoperative hepatocellular carcinoma: a systematic review. Int J Clin Pract 66: 21-27, 2012.

8. Toh U, Fujii T, Mishima M, et al: Conventional chemotherapy combined with the repetitive immune cell transfer for patients with refractory advanced gastric cancer. Gan To Kagaku Ryoho 34: 1931-1933, 2007 (In Japanese)

9. Linn YC and Hui KM: Cytokine-induced NK-like T cells: from bench to bedside. J Biomed Biotechnol 2010: 435745, 2010.

10. Sangiolo D: Cytokine induced killer cells as promising immunotherapy for solid tumors. J Cancer 2: 363-368, 2011.

11. Ma Y, Zhang Z, Tang L, et al: Cytokine-induced killer cells in the treatment of patients with solid carcinomas: a systematic review and pooled analysis. Cytotherapy 14: 483-493, 2012.

12. Wright SE, Rewers-Felkins KA, Quinlin IS, et al: Cytotoxic T-lymphocyte immunotherapy for ovarian cancer: a pilot study. J Immunother 35: 196-204, 2012.

13. Hickey MJ, Malone CC, Erickson KE, et al: Implementing preclinical study findings to protocol design: translational studies with alloreactive CTL for gliomas. Am J Transl Res 4: 114-126, 2012.

14. Yang M, Baranov E, Jiang P, et al: Whole-body optical imaging of green fluorescent protein-expressing tumors and metastases. Proc Natl Acad Sci USA 97: 1206-1211, 2000.

15. Chishima T, Miyagi Y, Wang X, et al: Cancer invasion and micrometastasis visualized in live tissue by green fluorescent protein expression. Cancer Res 57: 2042-2047, 1997.

16. Heim R, Cubitt AB and Tsien RY: Improved green fluorescence. Nature 373: 663-664, 1995.

17. Kaneko K, Yano M, Tsujinaka T, et al: Establishment of a visible peritoneal micrometastatic model from a gastric adenocarcinoma cell line by green fluorescent protein. Int J Oncol 16: 893-898, 2000.

18. Kaneko K, Yano M, Yamano T, et al: Detection of peritoneal micrometastases of gastric carcinoma with green fluorescent protein and carcinoembryonic antigen promoter. Cancer Res 61: 5570-5574, 2001.

19. Schmidt-Wolf IG, Negrin RS, Kiem HP, Blume KG and Weissman IL: Use of a SCID mouse/human lymphoma model to evaluate cytokine-induced killer cells with potent antitumor cell activity. J Exp Med 174: 139-149, 1991.

20. Linn YC, Lau LC and Hui KM: Generation of cytokine-induced killer cells from leukaemic samples with in vitro cytotoxicity against autologous and allogeneic leukaemic blasts. $\mathrm{Br} J$ Haematol 116: 78-86, 2002.

21. Lopez RD, Waller EK, Lu PH and Negrin RS: CD58/LFA-3 and IL-12 provided by activated monocytes are critical in the in vitro expansion of $\mathrm{CD}^{2} 6^{+} \mathrm{T}$ cells. Cancer Immunol Immunother 49: 629-640, 2001
22. Niam M, Linn YC, Fook Chong S, et al: Clinical scale expansion of cytokine-induced killer cells is feasible from healthy donors and patients with acute and chronic myeloid leukemia at various stages of therapy. Exp Hematol 39: 897-903, 2011.

23. Hazelrigg MR, Hirsch JI and Merchant RE: Distribution of adoptively transferred, tumor-sensitized lymphocytes in the glioma-bearing rat. J Neurooncol 60: 143-150, 2002.

24. Skitzki J, Craig RA, Okuyama R, et al: Donor cell cycling, trafficking, and accumulation during adoptive immunotherapy for murine lung metastases. Cancer Res 64: 2183-2191, 2004.

25. Edinger M, Cao YA, Verneris MR, Bachmann $\mathrm{MH}$, Contag $\mathrm{CH}$ and Negrin RS: Revealing lymphoma growth and the efficacy of immune cell therapies using in vivo bioluminescence imaging. Blood 101: 640-648, 2003.

26. Nishimura R, Baker J, Beilhack A, et al: In vivo trafficking and survival of cytokine-induced killer cells resulting in minimal GVHD with retention of antitumor activity. Blood 112: 2563-2574, 2008.

27. Brandau S and Bohle A: Activation of natural killer cells by Bacillus Calmette-Guerin. Eur Urol 39: 518-524, 2001.

28. Akagi J, Takai E, Tamori Y and Ogawa M: $\mathrm{CD} 3^{+} \mathrm{CD} 56^{+} \mathrm{CD} 8^{+}$cells demonstrating a suppressor $\mathrm{T}$ cell-like function in the peripheral blood of colon cancer patients. Int J Oncol 19: 561-566, 2001.

29. Nagaraj S, Ziske C and Schmidt-Wolf IG: Human cytokineinduced killer cells have enhanced in vitro cytolytic activity via non-viral interleukin-2 gene transfer. Genet Vaccines Ther 2: 12 , 2004.

30. Marten A, Renoth S, von Lilienfeld-Toal M, et al: Enhanced lytic activity of cytokine-induced killer cells against multiple myeloma cells after co-culture with idiotype-pulsed dendritic cells. Haematologica 86: 1029-1037, 2001.

31. Ziske C, Marten A, Schottker B, et al: Resistance of pancreatic carcinoma cells is reversed by coculturing NK-like T cells with dendritic cells pulsed with tumor-derived RNA and CA 19-9. Mol Ther 3: 54-60, 2001.

32. van Spriel AB, van Ojik $\mathrm{HH}$ and van De Winkel JG: Immunotherapeutic perspective for bispecific antibodies. Immunol Today 21: 391-397, 2000

33. Flieger D, Kufer P, Beier I, Sauerbruch T and Schmidt-Wolf IG: A bispecific single-chain antibody directed against EpCAM/ CD3 in combination with the cytokines interferon alpha and interleukin-2 efficiently retargets $\mathrm{T}$ and $\mathrm{CD}^{+} \mathrm{CD}^{+} 6^{+}$naturalkiller-like T lymphocytes to EpCAM-expressing tumor cells. Cancer Immunol Immunother 49: 441-448, 2000.

34. Lutterbuese R, Raum T, Kischel R, et al: Potent control of tumor growth by CEA/CD3-bispecific single-chain antibody constructs that are not competitively inhibited by soluble CEA. J Immunother 32: 341-352, 2009.

35. Savage P, Millrain M, Dimakou S, Stebbing J and Dyson J: Expansion of $\mathrm{CD}^{+}$cytotoxic $\mathrm{T}$ cells in vitro and in vivo using MHC class I tetramers. Tumour Biol 28: 70-76, 2007.

36. Morishima N, Mizoguchi I, Okumura M, et al: A pivotal role for interleukin-27 in $\mathrm{CD}^{+} \mathrm{T}$ cell functions and generation of cytotoxic T lymphocytes. J Biomed Biotechnol 2010: 605483, 2010.

37. Yee C, Thompson JA, Byrd D, et al: Adoptive T cell therapy using antigen-specific $\mathrm{CD} 8^{+} \mathrm{T}$ cell clones for the treatment of patients with metastatic melanoma: in vivo persistence, migration, and antitumor effect of transferred T cells. Proc Natl Acad Sci USA 99: 16168-16173, 2002

38. Mackensen A, Meidenbauer N, Vogl S, Laumer M, Berger J and Andreesen R: Phase I study of adoptive T-cell therapy using antigen-specific $\mathrm{CD} 8^{+} \mathrm{T}$ cells for the treatment of patients with metastatic melanoma. J Clin Oncol 24: 5060-5069, 2006.

39. Butler MO, Lee JS, Ansen S, et al: Long-lived antitumor CD8 ${ }^{+}$ lymphocytes for adoptive therapy generated using an artificial antigen-presenting cell. Clin Cancer Res 13: 1857-1867, 2007.

40. Yamaguchi Y, Ohshita A, Hironaka K, et al: Adoptive immunotherapy using autologous lymphocytes sensitized with HLA class I-matched allogeneic tumor cells. Oncol Rep 16: 165-169, 2006. 